\title{
Co-Expression of VEGF-C and Its Receptors, VEGFR-2 and VEGFR-3, in Endothelial Cells of Lymphangioma. Implication in Autocrine or Paracrine Regulation of Lymphangioma
}

\author{
Hsin-Yi Huang, Chao-Chi Ho, Pei-Hsin Huang, and Su-Ming Hsu \\ Department of Pathology, National Taiwan University College of Medicine and National Taiwan University Hospital, \\ Taipei, Taiwan
}

\begin{abstract}
SUMMARY: Lymphangioma has long been thought of as congenital malformations resulting from the failure of lymphatic vessels communicating with the venous system in the fetal period. Alternatively, it is proposed to be true neoplasm originated from the transformation of lymphatic endothelia. To extend the molecular basis of the pathogenesis of lymphangioma, we have characterized the expression of vascular endothelial growth factor (VEGF) and VEGF receptors (VEGFR) in 29 cases of lymphangioma by RNA in situ hybridization. Endothelial cells of lymphangioma co-express transcripts of VEGF-C and its receptors VEGFR-3 (Flt4) and VEGFR-2 (Flk1), which are not detectable in the adjacent connective tissue. In contrast, there is little or no expression of VEGF-C, VEGFR-3, and VEGFR-2 mRNA in endothelial cells of hemangiomas, angiosarcomas, or normal lymphatic vessels of the small or large intestines. The results suggest that VEGF-C and its receptors may take active parts in the formation of lymphangioma by autocrine or paracrine regulation. (Lab Invest 2001, 81:1729-1734).
\end{abstract}

\begin{abstract}
ymphangiomas are the most common human L lymphatic disorders. Histologically, they consist of large lymphatic channels growing in loose connective tissue stroma, which may contain collections of lymphocytes. Disorganized bundles of smooth muscle can be present in the lymphatic walls. Based on the fact that lymphatic channels sprout from the venous system during embryogenesis, lymphangiomas are thought of as a congenital lesion resulting from the failure of the lymphatic system to communicate with the venous system at the fetal period. On the contrary, it is argued that instead of being a congenital malformation, lymphangioma is a true neoplasm resulting from transformed lymphatic endothelial cells and/or stromal cells (Enzinger and Weiss, 1995).

The recently identified vascular endothelial growth factor C (VEGF-C) belongs to the plateletderived growth factor (PDGF)/VEGF family of growth factors and is a ligand for the endothelial-specific receptor tyrosine kinases VEGF receptor 3 (VEGFR-3) and VEGFR-2. VEGF-C, initially purified from the culture medium conditioned by $\mathrm{PC}-3$ prostatic adenocarcinoma cells, consists of two proteo-
\end{abstract}

Received September 27, 2001.

This work was supported in part by an internal grant for scientific research from National Taiwan University Hospital, grant number 000-20-1026. Address reprint requests to: Dr. Pei-Hsin Huang, Department of Pathology, National Taiwan University Hospital, 7 Chung-Shan S. Road, Taipei, Taiwan.E-mail:passion@ha.mc.ntu.edu.tw lytically processed polypeptides of 29 and $31 \mathrm{kD}$. It forms homodimers and induces tyrosine phosphorylation of the receptors VEGFR-2 and -3 (Galland et al, 1993; Pajusola et al, 1994). During mouse embryogenesis, VEGF-C mRNA is specifically expressed in the mesenchymal cells of the regions where the lymphatic vessels sprout from the venous system, whereas the expression of VEGFR-3, the VEGF-C receptor, is restricted in lymphatic endothelia. Such expression pattern of VEGF-C and its receptor VEGFR-3 suggests a paracrine mode of action in the regulation of lymphangiogenesis (Kukk et al, 1996). Moreover, VEGF-C transgenic mice display selective hyperplasia of the lymphatic vasculature, indicating that VEGF-C may play a role in disorders involving the lymphatic system (Jeltsch et al, 1997).

In this study, we explore the possible pathophysiological role of VEGF family of growth factors and their cognate receptors in lymphangioma. We have examined the expression pattern of VEGF subtypes and its receptors in lymphangioma by RNA in situ hybridization. Interestingly, we found that transcripts of VEGF-C, as well as its receptor VEGFR-3 and VEGFR-2, are co-localized in the lymphatic endothelial cells, whereas the mesenchymal cells of lymphangioma expressed little detectable transcripts of VEGF or VEGFR. Paracrine or autocrine regulation of VEGF-C and its cognate receptor is, thus, proposed to take an active role in the formation of lymphangioma. 


\section{Results}

Ectopic Expression of VEGF-C Transcripts in Endothelial Cells, but not in Mesenchymal Cells of Lymphangioma

The ligand of VEGFR-3, VEGF-C, was expressed in mesenchymal cells adjacent to the VEGFR-3-expressing lymphatic endothelia during embryogenesis and in adult tissues. Therefore, it is reasonable to expect that VEGF-C might be expressed in the mesenchymal cells of lymphangioma if it did play some role in the formation of lymphangioma. To our surprise, VEGF-C transcripts were clearly present in the cytoplasm of lymphatic endothelial cells but not in the mesenchymal cells of lymphangiomas (Fig. 1B, arrows). Although the intensity of the hybridization signal varied from case to case, it was always detectable in the endothelial cells. In contrast, there was no labeling in the connective tissue stroma and in lymphocytes (Fig. 1B). In addition, VEGF-C transcripts were not detected in any type of cell in cases of hemangioma or angiosarcoma (Fig. 1F and $1 \mathrm{~J})$, indicating that VEGF-C was indeed a specific marker for lymphatic system, not only during embryogenesis, but also in the lymphatic-derived pathological lesions such as lymphangioma. To our notice, the lymphatic vessels in the submucosa of normal large intestines did not show significant positive labeling for mRNAs of VEGF-C or its receptors VEGFR-2 and VEGFR-3 (data not shown). These results suggest to us that ectopic expression of VEGF-C in endothelial cells underlies the pathogenesis of lymphangioma.

\section{VEGFR-2 and VEGFR-3, the Receptors of VEGF-C, Localized to the Endothelial Cells of Lymphangioma as in the Expression Pattern of VEGF-C}

To assess the expression pattern of the VEGF-C cognate receptors VEGFR-2 and VEGFR-3 in comparison with that of VEGF-C in lymphangiomas, RNA in situ hybridization was performed. Continuous sections of $5-\mu \mathrm{m}$ thickness for each case were used to facilitate the comparison of in situ results from the different probe. As shown in Figure 1C and 1D, transcripts of VEGFR-2 and VEGFR-3 were seen in the endothelial cells of lymphangioma, and it was previously reported to be localized in the normal developing lymphatic endothelial cells during embryogenesis. Therefore, the transcripts of VEGF-C and its receptors VEGFR-2 and VEGFR-3, were co-localized to the endothelial cells of lymphangioma. For the control group of six cases of hemangioma and seven cases of angiosarcoma, we found that neither VEGFR-2 nor VEGFR-3 was expressed in the vascular endothelial cells (Fig. 1G, 1H, 1K, and 1L). The lymphatic vessels in the submucosa of the normal large intestine did not show significant positivity for mRNAs of VEGFR-2 and VEGFR-3 (data not shown).

\section{IL-1ß, which Stimulates the Transcription of VEGF-C and VEGFR-2, was Not Detectable in Lymphangioma Lesions by Immunohistochemistry}

$\mathrm{IL}-1 \beta$, a proinflammatory cytokine, induces a concentration- and time-dependent increase in VEGF-C
mRNA steady-state levels in human lung fibroblast. $\mathrm{IL}-1 \beta$ also can stimulate the transcription of VEGFR-2, but has no effect on VEGFR-3 (Ristimaki et al, 1998). Since VEGF-C was aberrantly expressed in the endothelial cells, which also expressed VEGFR-2 and -3, we explored the possibility of transcriptional co-regulation of VEGF-C and VEGFR-2 by IL-1 $\beta$. An immunohistochemical study was performed but no obvious staining was seen for lymphangioma. Such a result was comparable with the relative absence of inflammatory cell infiltrate in lymphangioma observed by hematoxylin and eosin stain. However, the possibility that circulating inflammatory cells secret IL-1 $\beta$ during the formation of lymphangiomas can still not be ruled out.

\section{Discussion}

The most widely accepted theory concerning the origin of the lymphatic system suggests that the lymphatic structure sprouts from large central veins in certain locations to form primordial lymph sacs during the fetal period. Later, the sacs enlarge, coalesce, and form new sprouts, which grow into the periphery of the embryo. The most common lesion of the lymphatic system is lymphangioma. It is usually seen on young patients and the most common localization of lymphangioma is in the head and neck region, especially at the posterior triangle of the cervical lateral region (Enzinger and Weiss, 1995). The age of onset and its preference location in the neck suggest a disembryogenetic pathogenesis of lymphangiomas. Alternatively, lymphangiomas are suspected as true tumors resulting from hyperplasia of transformed lymphatic endothelia caused by aberrant regulation of growth- or differentiation-related genes.

Recently, the PDGF/NEGF families, which are essential for vasculogenesis and/or angiogenesis, were expanded. Thus far, six members are known, which include PDGF, placenta growth factor (PIGF), VEGF, VEGF-B, VEGF-C, and VEGF-D (Achen et al, 1998; Joukov et al, 1996, 1997; Keck et al, 1989; Maglione et al, 1991; Olofsson et al, 1996; Paavonen et al, 1996; Yamada et al, 1997). PDGF is a major mitogen for connective tissue cells and certain other cell types (Heldin and Westermark, 1999), whereas the other five members specifically regulate the growth of endothelial cells in concert with their cognate receptors. Among these VEGF subtypes, VEGF-C is considered as a lymphatic endothelial-specific growth factor. During mouse embryogenesis, the cognate receptor of VEGF-C, VEGFR-3, becomes detectable in 8.5-day post coitum (p.c.) embryos in the angioblasts of head mesenchyme, the cardinal vein, and the allantois. In 12.5-day p.c. embryos, the VEGFR-3 mRNA is differentially expressed in developing venous and presumptive lymphatic endothelia but not in arterial endothelia. In the later stages of development, VEGFR-3 mRNA is restricted to developing lymphatic vessels (Kaipainen et al, 1995; Kukk et al, 1996). Consistent with its cognate receptor, VEGF-C was detected in mesenchymal cells with the similar temporal and spatial pattern of VEGFR-3. Moreover, overexpression of VEGF-C has been shown to selec- 

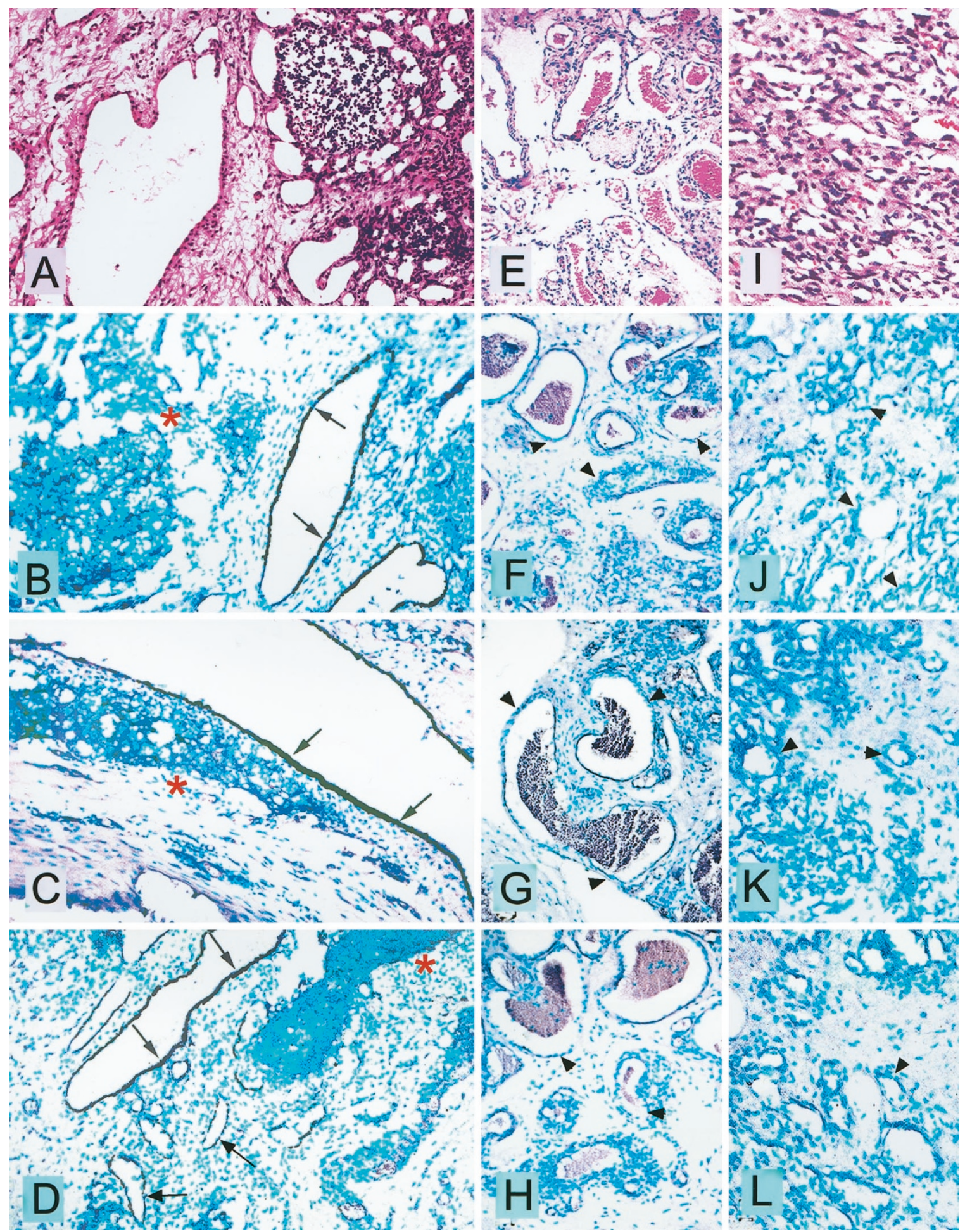

Figure 1.

RNA in situ hybridization showed co-expression of vascular endothelial growth factor-C (VEGF-C), VEGF receptor-2 (VEGFR-2), and VEGFR-3 in lymphangioma but not in hemangioma and angiosarcoma. A-D, Lymphangioma. A, H\&E stain. B, VEGF-C transcripts present in the endothelial cells (arrows) but not in the stromal cells or associated lymphocytes (indicated as red stars). C, VEGFR-2 transcripts also present in the endothelial cells (arrows) but not in the stroma. D, expression pattern of VEGFR-3 transcripts similar to that of VEGF-C and VEGFR-2. E-H, Hemangioma. I-L, Angiosarcoma. E and I, H\&E stain. Transcripts of VEGF-C (F and J), VEGFR-2 ( $G$ and $K)$, and VEGFR-3 ( $\mathrm{H}$ and $\mathrm{L}$ ) were not detected on the endothelial cells (arrowhead). Original magnification, $\times 200(\mathrm{~A}-\mathrm{L})$.

tively induce lymphatic, but not vascular, endothelial proliferation and hyperplasia of the lymphatic vasculature. These observations suggest a paracrine mode of ligand-receptor interaction for lymphatic growth, with
VEGF-C expressed in mesenchymal cells adjacent to the VEGFR-3-expressing lymphatic endothelia.

In normal human adult tissue, VEGF-C is expressed mainly in the heart, the placenta, the ovary, the small 
intestine, and the thyroid gland, suggesting that VEGF-C is also involved in the maintenance of functions of differentiated lymphatic endothelia by a paracrine fashion (Joukov et al, 1996). Increased expression of VEGFR-3 has also been reported to occur in the lymphatic sinuses of lymph nodes with metastatic carcinomas and in lymphangiomas. VEGF-C could also express in tumor cells in some solid human tumors, such as breast cancers, squamous cell carcinomas, melanomas, and some kinds of sarcomas (Lymboussaki et al, 1998; Partanen et al, 1999; Salven et al, 1998). One may speculate that VEGF-C plays an important role in regulating the proliferation of lymphatics and, in some ways, influences lymphatic vascular metastases.

In this study, VEGF-C mRNA was detected in the lymphatic endothelia of lymphangioma but not in the lymphatic adjacent mesenchymal cells as predicted from its normal distribution pattern. The expression of VEGF-C is specific for lymphatic endothelial cells, because it was not detectable in the mesenchymal cells or other vascular neoplasm including hemangioma and angiosarcoma, as well as the normal vascular endothelia of arteries, veins, and capillaries in adult tissue. In parallel with the expression pattern of VEGF-C in lymphangioma, the VEGF-C receptor, VEGFR-2, and VEGFR-3 were also expressed in the lymphatic endothelia. The expression of VEGFR-3 is more specific for lymphatic endothelial cells than that of VEGFR-2, because transcripts of VEGF-R2, but not of VEGFR-3, were also seen in the vascular endothelial cells of arteries, veins, and capillaries. Our finding is in agreement with other reports, which found that VEGFR-3 may be used as a marker for lymphatic endothelia, whether they are present in normal tissues or in lymphangioma. Moreover, the specific expression of VEGF-C in the endothelial cells of lymphangioma, but not in the normal lymphatic channels or other angiogenic lesions, suggests that VEGF-C alone could be a disease marker for lesions of lymphangioma.

The co-localization of VEGF-C and its receptors VEGFR-2 and -3 indicates that an autocrine or paracrine mode of interaction between VEGF-C and its receptors accounts for the formation of lymphangioma. In either condition, the acquisition of VEGF-C expression may play a critical role in the pathogenesis of lymphangioma. The autocrine/paracrine mode of VEGF-C and VEGFR-3 interaction could lead to an aberrant proliferation of transformed endothelial cells in lymphangiomas. With the expression of VEGFR-3, the endothelial cells in these tumors differentiate morphologically toward lymphatic vessels. Meanwhile, these cells secrete VEGF-C to maintain the proliferation and differentiation of endothelial cells. Based on the autocrine/paracrine model of VEGF-C, the growth and progression of lymphangioma are more likely neoplastic in nature rather than purely malformations.

Why endothelial cells in lymphangioma aberrantly expressed VEGF-C is not known. Although there is report that IL-1 $\beta$ could up-regulate the transcription of VEGF-C and VEGFR-2 (Ristimaki et al, 1998), it is difficult for us to make any conclusion about the status
IL-1 $\beta$ in lymphangioma by immunohistochemical examination only. The mechanism responsible for VEGF-C gene expression in lymphatic endothelial cells of lymphangioma remains to be elucidated.

\section{Materials and Methods}

\section{Specimens}

A total of 29 cases of lymphangioma, six cases of hemangioma, seven cases of angiosarcomas, and two cases of normal large intestine were collected from National Taiwan University Hospital from 1995 to 2000. The 29 cases of lymphangioma ranged in age from 2 months to 62 years. Thirteen patients were less than 1 year old at the time of diagnosis, and 11 patients ranged from 2 years old to 11 years old. The five patients diagnosed in adulthood were 36, 37, 47, 52 and 62 years old, respectively. The six patients with capillary and cavernous hemangioma ranged from 9 years old to 59 years old. Two of them are children, 9 and 12 years old. The seven patients of angiosarcoma were all in their seventh or eighth decades. All patients with lymphangioma and hemangioma, although some were diagnosed in the adulthood, were considered to have congenital lesions, because the chart history recorded that the lesions were diagnosed in their childhood. Those lesions with dilated lymphatic channels secondary to obstruction of draining lymphatic ducts, such as those seen on the arms after radiotherapy for breast cancer, were not included in this study. Each was selected from archival formalin-fixed, paraffin-embedded blocks stored in the Department of Pathology, National Taiwan University Hospital, Taipei, Taiwan. Eighty-seven children with lymphangioma were identified over a 5.5-year period at our hospital. This number is estimated to account for about $0.35 \%$ of pediatric admission cases. The incidence is roughly equal for both sexes.

\section{Light Microscopy}

All cases were reviewed with a light microscope for diagnosis (Leica DMRD; Leica GmbH, Wetzlar, Germany). By hematoxylin- and eosin-stain (H\&E stain), lymphangiomas showed characteristic large lymphatic channels lined by a layer of flattened endothelial cells in a loose connective tissue stroma. Focal collections of lymphocytes were occasionally seen. The cases of hemangioma consisted of dilated or small vascular spaces lined by endothelial cells.

\section{RNA Extraction and RT-PCR}

Total RNA was extracted from the freshly frozen human placental tissue with Trizol reagent and was reverse-transcribed to complementary DNA (Gibco BRL, Rockville, Maryland). PCR was performed with the following primers and condition. For VEGF-C, the primers were 5'-TTGCCAATCACACTTCCTGCC GATGCATGT-3' (upstream) and 5'-ACTATATGAAAATCCTGGCTCACAAGCCT-3' (downstream). PCR was performed at $55^{\circ}$ to $60^{\circ} \mathrm{C}$ (touchdown program) for 10 
cycles and then for an additional 20 cycles at $55^{\circ} \mathrm{C}$. For VEGFR-3, the primers were 5'-AACAACGGCATCCAGCGAT TT-3' (upstream) and 5'-AGATGCTCGTACTTGTAGCT-3' (downstream). PCR was performed at $45^{\circ} \mathrm{C}$ for 35 cycles. For VEGFR-2, the upstream and downstream primers were 5'- CAAGATAC CCAGAAAAGAGATTT-3' (upstream) and 5'CTTCCATAATCGTCAGTACAT-3' (downstream). PCR was done at $50^{\circ}$ to $55^{\circ} \mathrm{C}$ (touchdown program) for 10 cycles and at $50^{\circ} \mathrm{C}$ for another 25 cycles.

\section{Cloning and Riboprobe Synthesis}

DNA fragments of 300 to $600 \mathrm{bp}$ in length were eluted from the $1 \%$-agarose gel and ligated to $\mathrm{PCR} \| \mathrm{I}$ vector (Invitrogen, San Diego, California). After transformation and plasmid DNA purification, sequences were confirmed by autocycling sequencing (PerkinElmer ABI 373; PerkinElmer-Applied Biosystems, Foster City, California). Using these confirmed DNA fragments as templates, single-stranded complementary (antisense probes) and anticomplementary (sense probes) RNA probes were synthesized by in vitro transcription with T7-and Sp6-RNA polymerases using the DIG RNA transcription kit (Roche Molecular Biochemicals, Mannheim, Germany).

\section{In Situ Hybridization}

RNA in situ hybridization was performed on 10 to 12 $\mu \mathrm{m}$-thick formalin-fixed, paraffin-embedded tissue sections. Tissue sections were dewaxed through $\mathrm{xy}-$ lene, rehydrated with graded alcohol, treated with proteinase $\mathrm{K}$ ( $8 \mu \mathrm{g} / \mathrm{ml}$ for 3 minutes), acetylated, fixed in $4 \%$ paraformaldehyde, and then dehydrated with graded alcohol. Hybridization with Dig-labeled riboprobes was carried out at $58^{\circ} \mathrm{C}$ for 35 to 40 hours. Post-hybridization washes were performed several times at $50^{\circ} \mathrm{C}$. Anti-digoxigenin monoclonal antibody (Roche Molecular Biochemicals) was applied at the titer of 1:2000 in tris-buffered saline (TBST, pH 7.4, with $+0.1 \%$ Tween-20) at $4^{\circ} \mathrm{C}$ overnight. The slides were then washed for four times in TBST for 15 minutes each time, and three times in NTMT solution (100 mM NaCl, $100 \mathrm{~mm}$ Tris- $\mathrm{HCl}, 50 \mathrm{~mm} \mathrm{MgCl}_{2},+0.1 \%$ Tween-20, $2 \mathrm{~mm}$ levimasole, $\mathrm{pH}$ 9.5) for 10 minutes each time. Color development was achieved by layering freshly prepared substrate solution $(330 \mu \mathrm{g} / \mathrm{ml}$ 5-bromo-4-chloro-3-indolyl phosphate disodium salt and $165 \mu \mathrm{g} / \mathrm{ml}$ nitroblue tetrazolium salt in NTMT solution) to the slides for 16 to 20 hours. The tissue sections were counter-stained with methyl green and observed by light microscopy with H\&E-stained tissue sections as morphological comparisons.

\section{References}

Achen MG, Jeltsch M, Kukk E, Makinen T, Vitali A, Wilks AF, Alitalo K, and Stacker SA (1998). Vascular endothelial growth factor $D$ (VEGF-D) is a ligand for the tyrosine kinase receptor VEGF receptor 2 (Flk1) and VEGF receptor 3 (Flt4). Proc Natl Acad Sci USA 95:548-553.
Enzinger FM and Weiss SW (1995). Tumors of lymph vessels. In: Enzinger FM and Weiss SW, editors. Soft tissue tumors. St. Louis: Mosby-Year Book, 679-688.

Galland F, Karamysheva A, Pebusque MJ, Borg JP, Rottapel R, Dubreuil P, Rosnet O, and Birnbaum D (1993). The FLT4 gene encodes a transmembrane tyrosine kinase related to the vascular endothelial growth factor receptor. Oncogene 8:1233-1240.

Heldin CH and Westermark B (1999). Mechanism of action and in vivo role of platelet-derived growth factor. Physiol Rev 79:1283-1316.

Jeltsch M, Kaipainen A, Joukov V, Meng X, Lakso M, Rauvala H, Swartz M, Fukumura D, Jain RK, and Alitalo K (1997). Hyperplasia of lymphatic vessels in VEGF-C transgenic mice. Science 276:1423-1425.

Joukov V, Kaipainen A, Jeltsch M, Pajusola K, Olofsson B, Kumar V, and Alitalo K (1997). Vascular endothelial growth factors VEGF-B and VEGF-C. J Cell Physiol 173:211-215.

Joukov V, Pajusola K, Kaipainen A, Chilov D, Lahtinen I, Kukk E, Saksela O, Kalkkinen N, and Alitalo K (1996). A novel vascular endothelial growth factor, VEGF-C, is a ligand for the Flt4 (VEGFR-3) and KDR (VEGFR-2) receptor tyrosine kinases. EMBO J 15:209-298.

Kaipainen A, Korhonen J, Mustonen T, van Hinbergh VW, Fang GH, Dumont D, Breitman M, and Alitalo K (1995). Expression of the fms-like tyrosine kinase 4 gene becomes restricted to lymphatic endothelium during development. Proc Natl Acad Sci USA 92:3566-3570.

Keck PJ, Hauser SD, Krivi G, Sanzo K, Warren T, Feder J, and Connolly DT (1989). Vascular permeability factor, an endothelial cell mitogen related to PDGF. Science 246:13091312.

Kukk E, Lymboussaki A, Taira S, Kaipainen A, Jeltsch M, Joukov V, and Alitalo K (1996). VEGF-C receptor binding and pattern of expression with VEGFR-3 suggests a role in lymphatic vascular development. Development 122:38293837.

Lymboussaki A, Partanen TA, Olofsson B, Thomas-Crussels J, Fletcher CDM, de Waal RMW, Kaipainen A, and Alitalo K (1998). Expression of the vascular endothelial growth factor $\mathrm{C}$ receptor VEGFR-3 in lymphatic endothelium of the skin and in vascular tumors. Am J Path 153:395-403.

Maglione D, Guerriero V, Viglietto G, Delli-Bovi P, and Persoco MG (1991). Isolation of a human placenta cDNA coding for a protein related to the vascular permeability factor. Proc Natl Acad Sci USA 88:9267-9271.

Olofsson B, Pajusola K, Kaipainen A, von Euler G, Joukov V, Saksela O, Orpana A, Pettersson RF, Alitalo K, and Eriksson U (1996). Vascular endothelial growth factor B, a novel growth factor for endothelial cells. Proc Natl Acad Sci USA 93:2376-2381.

Paavonen K, Horelli Kuitunen N, Chilov D, Kukk E, Pennanen S, Kallioniemi OP, Paavonen K, Olofsson B, Eriksson U, Joukov V, Palotie K, Joukov V, and Alitalo K (1996). Novel human vascular endothelial growth factor genes VEGF-B and VEGF-C localize to chromosome $11 \mathrm{q} 13$ and $4 \mathrm{q} 34$, respectively. Circulation 93:1079-1082.

Pajusola K, Aprelikova O, Pelicci G, Weich H, ClaessonWelsh L, and Alitalo K (1994). Signalling properties of FLT4, a proteolytically processed receptor tyrosine kinase related to two VEGF receptors. Oncogene 9:3545-3555. 
Partanen TA, Alitalo K, and Miettinen M (1999). Lack of lymphatic vascular specificity of vascular endothelial growth factor receptor 3 in 185 vascular tumors. Cancer 86:24062412.

Ristimaki A, Narko K, Enholm B, Joukov V, and Alitalo K (1998). Proinflammatory cytokines regulate expression of the lymphatic endothelial mitogen vascular endothelial growth factor-C. J Biol Chem 273:8413-8418.
Salven P, Lymboussaki A, Heikkila P, Jaaskela-Saari H, Enholm B, Aase K, von Euler G, Eriksson U, Alitalo K, and Joensuu H (1998). Vascular endothelial growth factors VEGF-B and VEGF-C are expressed in human tumors. Am J Pathol 153:103-108.

Yamada Y, Nezu J, Shimane M, and Hirata Y (1997). Molecular cloning of a novel vascular endothelial growth factor, VEGF-D. Genomics 42:483-488. 